\title{
Diagnóstico y tratamiento de pacientes COVID-19 en Urgencia Pediátrica. Guía para esta pandemia
}

\author{
Diagnosis and treatment of pediatric patients with COVID-19 infectious \\ disease at the emergency room. A guideline for this pandemia \\ Ida Concha ${ }^{a}$, Carlos Fernández ${ }^{\mathrm{b}}$, Tamara Hirsch $^{\mathrm{a}}$, Francisco Prado ${ }^{\mathrm{c}}$, Viera Morales $^{\mathrm{a}}$, Astrid Pezoa $^{\mathrm{a}}$
}

\author{
aServicio de Urgencia, Hospital Clínico Pontificia Universidad Católica de Chile, División de Pediatría, Facultad de Medicina, Pontificia Universidad \\ Católica de Chile. Santiago, Chile \\ bServicio de Urgencia, Clínica Santa María. Santiago, Chile \\ 'Servicio de Pediatría, Hospital Clínico San Borja Arriarán, Departamento de Pediatría y Cirugía Infantil, Campus Centro, Facultad de Medicina, \\ Universidad de Chile. Santiago, Chile
}

Recibido: 3 de mayo de 2020; Aceptado para publicación: 18 de mayo de 2020

\begin{abstract}
Resumen
La emergencia del virus SARS-CoV-2 (CoV-2), ha producido nuevos y grandes desafíos para la salud pública y la atención de urgencia desde su aparición en Wuhan, China ${ }^{1}$. Los desafíos en urgencia, van desde cambios en los flujos de pacientes, áreas de atención, sistemas de trabajo, equipo de protección personal (EPP) necesarios y una gran capacitación para lograr atender adecuadamente a adultos y niños con la enfermedad producida por este virus (COVID-19) $)^{2-4}$. En esta publicación, se propone una guía de cómo organizar un servicio de urgencias en tiempos de pandemia y cómo atender al niño sospechoso o con COVID-19 que consulta en urgencias. La información necesaria se obtuvo a través de guías nacionales e internacionales y la literatura científica obtenida a través de PubMed, CDC y NIH y de la autoridad sanitaria nacional.
\end{abstract}

\section{Abstract}

The SARS-CoV-2 emergency has caused major new challenges for both public health and medical clinical practice since the first cases reported in Wuhan, China. The challenges in emergency care range from changes in the flow of patients, care areas, ways of working, personal protective equipment, and intensive training in order to properly care for adults and children with coronavirus disease (COVID-19). In this publication, we propose guidelines on organizing the emergency department in the face of this pandemic, and how to provide medical care for the pediatric patient with either suspected or confirmed COVID-19 who seek care in the emergency room. We obtained the necessary data from national and international guidelines and the scientific literature available from PubMed, CDC, NIH, and the Chilean national health authorities.
Palabras clave:

SARS-CoV-2;

COVID-19;

Servicio de Urgencia Pediátrica

Keywords: SARS-CoV-2; COVID-19; Pediatric Emergency Room

Correspondencia:

Ida A. Concha M

iconcha@med.puc.cl 


\section{Introducción}

En la población pediátrica el virus $\mathrm{CoV}-2$ produce, afortunadamente, cuadros la mayoría de las veces leves pero también puede producir cuadros de mayor gravedad como neumonías con requerimientos de oxígeno que necesitarán hospitalización, manifestaciones extrapulmonares pleomórficas y otras menos frecuentes con requerimientos de cuidados intensivos por insuficiencia respiratoria aguda grave hipoxémica, síndrome de distress respiratorio agudo (SDRA), compromiso miocárdico y otras formas de síndrome inflamatorio multisistémico que pueden llevar al shock secundario, con un cuadro clínico similar a enfermedad de Kawasaki, síndrome de shock tóxico y síndrome de activación macrofágica (SAM) $)^{5,6}$.

Los niños, representan menos del 5\% del total de infectados, han sido reconocidos como importantes transmisores asintomáticos que pueden contagiar a personas adultas de mayor riesgo y seguir propagando la enfermedad. La organización de los servicios de urgencias es vital para tratar de aislar a los pacientes con COVID-19, de modo de evitar nuevos contagios. A continuación, se detallan las principales recomendaciones para la atención de estos enfermos en un servicio de urgencias.

\section{Definiciones}

Estas fueron dadas por el Ministerio de salud y han sido modificados según cómo ha evolucionado la pandemia $^{7}$.

\section{Caso sospechoso}

- Fiebre (temperatura sobre $37,8^{\circ} \mathrm{C}$ axilar) sin otra causa evidente.

- Síntomas sugerentes de infección respiratoria tales como mialgias, odinofagia, tos, disnea, dolor torácico, taquipnea, cianosis, postración, y/o dolor abdominal, diarrea.

- Alteración aguda del olfato (anosmia) o gusto (disgeusia).

\section{Caso confirmado COVID-19}

- Caso sospechoso con muestra positiva para CoV-2 por reacción en cadena de polimerasa con transcripción reversa en tiempo real (RT-PCR).

- Caso sospechoso e imágenes características en la tomografía computarizada (TAC) de tórax.

- Caso clínico típico y un resultado positivo en una prueba serológica (IgM, IgG o ambos).

\section{Infectado con SARS-CoV-2}

- Detectados por RT-PCR o por serología positiva en sujetos asintomáticos, durante una búsqueda activa o detectados por screening.

\section{Caso probable COVID-19}

- Persona que presenta un cuadro clínico compatible y que es contactos conocido de casos confirmados por laboratorio con prueba RT-PCR.

\section{Organización del flujo de pacientes: dos flujos separados $^{2-4}$}

Las recomendaciones en los flujos de urgencia han emanado fundamentalmente de expertos en infecciones intrahospitalarias, infectólogos, personal de SU y de la autoridad sanitaria.

1. Al ingreso del hospital debe existir un pre-triage donde esté un profesional que pregunte por síntomas sospechosos de COVID-19. Si los hay, el paciente tendrá un flujo totalmente distinto al paciente con otro motivo de consulta. Los casos más leves, se sugiere que sean referidos a un área acondicionada especialmente para la atención de paciente COVID-19 fuera del hospital. El resto de los pacientes irán a un área COVID-19 dentro del hospital, idealmente box aislado o camillas separadas al menos por 1 metro de distancia de otra.

2. La enfermera o técnico paramédico de triage instala una mascarilla quirúrgica al paciente y a su acompañante, lo evalúa y categoriza. Luego, es enviado al área correspondiente para su atención o a una sala de espera específica para problemas COVID-19, con separaciones de al menos un metro de otro paciente.

3. Para lograr evitar contagios, es necesario tener equipos sanitarios distintos para cada tipo de atención, es decir, técnicos, enfermeras y médicos que trabajen independientemente en cada área.

4. El personal de pre-triage y triage y todo el resto del personal que trabaje en urgencias debe estar protegido con mascarilla quirúrgica. Ésta debe usarse en todo momento, independiente si está en las salas sospechosas de COVID-19, y cada 4-6 h deberá cambiársela o antes si se humedece. Los estudios son claros en demostrar la capacidad de protección de la mascarilla quirúrgica contra el virus $\mathrm{CoV}-2$ en situaciones no generadoras de aerosoles ${ }^{8}$. Se ha descrito que alrededor del $30 \%$ de los profesionales de la salud se contagiaran', por lo que el cuidado es clave para evitar quedarse con personal insuficiente para la atención de los enfermos.

\section{Atención en box de paciente COVID-19}

Idealmente debe ser individual con puertas cerradas. Todo el personal que atiende a estos pacientes debe usar EPP de gotitas y de contacto (delantal de plástico, guantes, mascarilla quirúrgica). El médico que atenderá al paciente se aconseja que además utilice 
escudo facial, por el estrecho contacto ${ }^{10}$. Hay protocolos filmados para uso correcto de $\mathrm{EPP}^{11}$.

El box debe contar con un estetoscopio, otoscopio, conos desechables y bajalenguas. El médico determinará si cumple el criterio de caso sospechoso, si es así, indicará toma de RT-PCR para el diagnóstico de COVID-19 e indicará eventualmente otros exámenes según estime conveniente para un correcto tratamiento del paciente. Una vez dado de alta el box de atención requiere aseo terminal. Se ha descrito que el virus es capaz de quedar en los objetos inertes por largas horas por lo que es necesario limpiar todas las superficies con amonio cuaternario o alcohol isopropílico al 70\% o más ${ }^{12}$.

\section{Cuadro clínico}

Hasta el momento, la mayoría de los casos descritos en pediatría son leves, lo interesante es que, al contrario de los adultos infectados, menos del 50\% tienen fiebre y/o síntomas respiratorios. Es muy frecuente que sean asintomáticos, lo que dificulta el control de la propagación del virus. Desafortunadamente hay casos más serios que progresan hacia neumonías, inclusive algunos con necesidad de soporte de oxígeno, ventilación mecánica y apoyo intensivo ${ }^{13-16}$. Se han clasificado los síntomas en 4 categorías de gravedad. El médico de urgencias deberá determinar en qué nivel queda el paciente que está evaluando y según esto qué tratamiento requiere.

- Leve: Tienen un poco de tos seca, escasa rinorrea, estornudos, fiebre baja, malestar en general, mialgias y odinofagia. Al examen sólo habrá congestión faríngea y una auscultación pulmonar normal. Otros sólo tendrán náuseas, vómitos y diarrea.

- Moderado: Éstos tienen neumonía. Hay fiebre, tos seca que luego se hace productiva, al examen físico tienen crepitaciones, algunas sibilancias, pero no están polipneícos ni hipoxémicos. Otros no tienen síntomas ni signos clínicos, pero sí neumonía a la radiografía o scanner pulmonar.

- Grave: Con síntomas respiratorios, tos, fiebre desde el inicio y a la semana comienzan con disnea y dificultad respiratoria, saturación de oxígeno < 92\% y otros signos de hipoxemia.

- Crítico: Comienzan con síntomas respiratorios y rápidamente progresan a una insuficiencia respiratoria, SDRA asociado a shock, miocardiopatía, coagulopatía, encefalopatía, falla renal.

\section{Toma de exámenes}

- $\quad$ RT-PCR para CoV-2: Si el médico determina que el paciente es sospechoso de COVID-19 el personal de enfermería tomará el examen de RT-PCR para virus CoV-2 a través de un hisopado nasofaríngeo, con precauciones aún mayores que las descritas anteriormente, considerando que tendrá contacto con secreciones del paciente: precauciones de contacto y respiratorio con mascarilla N95 (filtro de alta eficiencia), gafas o antiparras, delantal desechable y guantes ${ }^{8-11}$. La toma de muestra del hisopado nasofaríngeo puede enviarse al Instituto de Salud Pública (ISP) con formulario ad/hoc con doble caja de protección o en los centros privados que cuentan con el examen certificado por el ISP, se toma éste. El resultado del examen estará en 1-2 días y se avisará si sale positivo. Se describe una sensibilidad de RT-PCR para CoV2 cercana al $80 \%{ }^{17}$.

- Exámenes de sangre: Se justifican en caso de pacientes que se hospitalizan, calificados como graves (neumonía con requerimientos de oxígeno), en pacientes con patología de base que puedan descompensarse a consecuencia de la infección. Para ellos, bastaría un hemograma completo, electrólitos plasmáticos, deshidrogenasa láctica, creatinina y nitrógeno ureico, procalcitonina si se sospecha sobreinfección bacteriana. Para los enfermos críticos, se debe agregar gases venosos, ferritina, dimero-D, creatin kinasa total y miocárdica, troponinas, y eventualmente procalcitonina ${ }^{16,18-20}$.

- Radiografía de tórax: Debe realizarse en casos en que haya sospecha de neumonía o en aquellos que sin tener auscultación alterada tienen polipnea, taquipnea o saturación de oxígeno baja. Los hallazgos más frecuentes son imágenes parenquimatosas e intersticiales tenues en las bases pulmonares y en los casos más severos imágenes de consolidación multilobar bilateral ${ }^{16,18,19}$.

- Scanner pulmonar (TAC): Aun cuando no está indicado rutinariamente en urgencias, puede ser de ayuda en aquellos pacientes más graves, con compromiso radiológico indeterminado o poco evidente. En las pocas series pediátricas que existen, el patrón en vidrio esmerilado de localización preferentemente en las bases pulmonares y región subpleural, junto con el patrón en empedrado se observa en más del $50 \%$ de los niños hospitalizados. Otro patrón, reconocible y sugerente, es el de condensación con halo circundante. Es decir, pequeños focos de consolidación a veces de aspecto pseudonodular, rodeados por vidrio esmerilado, dando cuenta del compromiso centrolobulillar, intersticio-alveolar parcial, de los estadios iniciales ${ }^{21,22}$.

- Ecografía pulmonar: Son pocas las descripciones que se han hecho en niños. El compromiso pleural con derrame y más aún con tabiques, excluye el diagnóstico de COVID-19. En adultos, y en servicio de urgencia se ha visto su rol en identificar el edema alveolo-intersticial concordante con el 
patrón en vidrio esmerilado y empedrado descrito en TAC. Podría ser de ayuda en niños categorizados en estado grave o crítico y que no pueden ser movilizados a una sala de rayos ${ }^{23}$.

\section{Alta a domicilio}

Los pacientes catalogados como leves o moderados sin factores de riesgo y mayores de 1 año pueden enviarse a casa. No requieren de nuevos controles a menos que tengan un quiebre en su sintomatología y aparezcan signos de progresión o complicación de la enfermedad ${ }^{19}$.

Pueden controlarse vía telemedicina para ver evolución del cuadro. El tratamiento es sólo sintomático y es fundamental explicar detenidamente los signos de alarma y las medidas de aislamiento e higiene para evitar nuevos contagiados en el grupo familiar.

\section{Aislamiento y medidas de higiene}

Los pacientes en que el examen de RT-PCR salga positivo deben mantenerse aislados por 14 días en casa o hasta que sus síntomas hayan desaparecido. Durante el tiempo de aislamiento, no puede haber visitas en casa.

El niño idealmente deberá permanecer en una pieza con baño individual. Si esto no es posible, mantener un distanciamiento de al menos un metro y realizar limpieza de baño antes que un tercero lo use, con amonio cuaternario o cloro al $0,1 \%$ ( $20 \mathrm{cc}$ de cloro doméstico con $1.000 \mathrm{cc}$ de agua).

Todo aquel que esté al cuidado del paciente o cercano a él debe portar una mascarilla quirúrgica. El lavado de manos será antes y después de estar con el niño, utilizar toalla exclusiva, idealmente secarse con toalla de papel. Las ropas se acumulan en bolsa y luego se lavan. Los platos y cubiertos deben ser exclusivos para los pacientes.

Los pañales deben ser puestos en bolsas para luego ser eliminados en basura, siempre manipularlos con guantes. Luego lavarse bien las manos.

El aseo de la pieza debe ser diario, con cloro o productos con amonio cuaternario ${ }^{24}$.

No está contraindicada la lactancia materna.

\section{Tratamiento de soporte}

El cuadro respiratorio o febril debe ser tratado en forma similar a cualquier otro paciente. Si hay fiebre, se aconseja uso de paracetamol como primera opción. Se observaron en Francia algunos casos de adultos que supuestamente habían tenido peor evolución con uso de antiinflamatorios no esteroidales, la causalidad no ha sido demostrada ${ }^{25}$. Si hay obstrucción bronquial deberá indicarse uso de inhalador dosis medida (IDM) con aerocámara individual. Deben mantenerse las medidas habituales de hidratación y realizar aseo nasal si es estrictamente necesario (cuidador siempre debe estar con mascarilla, y guantes en este procedimiento y lavarse las manos después de atender al niño).

\section{Tratamiento en urgencias del niño grave y crítico}

Según las diversas series clínicas de pacientes pediátricos extranjeras (Estados Unidos y China) los niños necesitan mucho menos hospitalización que los adultos, entre un 5,7-20\% y sólo entre el $0,58-2 \%$ en unidades de cuidados intensivos. Dentro de los más graves, el 77\% tenían una o más enfermedades de base como asma bronquial, enfermedades cardiovasculares e inmunodepresión. La mayoría de estos niños, eran hombres y más frecuentemente menores de un año ${ }^{14,26}$.

La valoración del paciente por el médico de urgencia es clave para lograr tratar a tiempo y adecuadamente a los más graves. Los parámetros que indican una mayor gravedad se indican en la tabla 1 .

A continuación, se analizan sucintamente las principales estrategias terapéuticas para el paciente con COVID-19 con mayor compromiso:

1. Monitoreo continuo o periódico de los signos vitales puesto que se han descrito cambios bruscos de la condición del paciente y hay que estar alerta a ellos.

2. Oxígeno para mantener saturación mayor o igual a $93 \%$.

Es consistentemente reconocido que la ventilación no invasiva (VMNI) y los sistemas de alto flujo por cánula nasal (Cnaf) son generadores de aerosoles y aumentan el riesgo de transmisión de patógenos ${ }^{27}$. Una simulación en maniquíes adultos con injuria pulmonar mostró que la máxima dispersión se producía con la ventilación no invasiva $(90 \mathrm{~cm}), y$ la menor con la mascarilla de no reinhalación (menos de $10 \mathrm{~cm}$ ). La cánula nasal, sistema amplia-

Tabla 1. Signos de gravedad en el paciente pediátrico con sospecha de COVID-19

- Frecuencia respiratoria

a) Menor de 2 meses: $\geq 60$ por minuto

b) 2 a 11 meses: $\quad \geq 50$ por minuto

c) 1 a 5 años: $\quad \geq 40$ por minuto

- Saturación de oxígeno $\leq$ o igual a 92\% (ambiental)

- Quejido, aleteo nasal, retracciones

- Cianosis

- Apneas

Deshidratación

- Compromiso de conciencia

Signos de shock (taquicardia, mala perfusión o hipotensión) 
mente utilizado en los pacientes con hipoxemia leve, presentó una dispersión de 40-30cm según los flujos usados ${ }^{28}$.

A nuestro entender, no existe información en pediatría que avale estos hallazgos, y lo aconsejable será tomar todas las precauciones disponibles antes de indicar un sistema altamente generador de aerosoles: utilizar siempre EPP, idealmente una sala a presión negativa y filtro HEPA (acrónimo del inglés High Efficiency Particular Air) en ramas espiratoria en caso de VMNI o en Cnaf.

3. Fluidos según necesidad: considerar que la volemización exagerada puede afectar aún más la función respiratoria y cardiovascular ${ }^{29}$.

4. Broncodilatadores por IDM y no por nebulización si hay obstrucción bronquial. Esto es por el riesgo que tienen las nebulizaciones de generar aerosoles que incrementen la propagación del virus ${ }^{27,28}$. La nebulización sólo puede ser realizada en una sala con presión negativa.

5. En caso de obstrucción de vía aérea alta con estridor grado III o IV, si no hay sala con presión negativa o una ventana al exterior en el box donde está el paciente, no se recomienda nebulizar con adrenalina racémica. Sugerimos utilizar adrenalina intramuscular en dosis similares a las de anafilaxia ${ }^{30}:<$ de

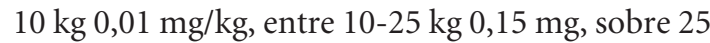
kg 0,3 mg. Estas recomendaciones están sólo basadas en la experiencia personal de los autores y en el efecto positivo reconocido que tiene la adrenalina en una vía aérea crítica como la anafilaxia. No es una manifestación frecuentemente descrita de COVID-19, pero puede caer en definición de MINSAL de caso sospechoso.

6. Corticoides: No tienen efectos beneficiosos, pueden retrasar la mejoría ${ }^{31}$. Podrían ser considerados en un SDRA ${ }^{31}$, frente a la aparición de encefalopatía, síndrome hemofagocítico o SAM, shock y en niños con asma bronquial.

7. Antibióticos: No están recomendados salvo en sospecha de sobreinfección bacteriana.

8. Azitromicina e hidroxicloroquina: Si bien existen protocolos para su uso en pacientes graves, hasta el momento no hay evidencia sólida para su uso en tratamientos ambulatorios ${ }^{32}$. Hidroxicloroquina tendría un efecto doble: impide la entrada del virus a la célula y modularía la respuesta inflamatoria que se ve en pacientes graves. Su uso no está exento de efectos colaterales y en pacientes con síndrome de QT largo puede producir arritmias severas.
9. Antivirales: Hasta ahora no se ha comprobado eficacia de antivirales en niños, no está recomendado su uso en urgencias.

10. Radiología: Si el paciente requiere una imagen, se debiera tomar con equipo portátil, para evitar traslados y nuevos contagios ${ }^{33}$. Si no es posible, realizar traslado con paciente utilizando mascarilla quirúrgica, el equipo de traslado con protección de contacto y de gotitas.

11. Intubación: En general no es recomendable realizarla en el servicio de urgencia, por los riesgos de generación de aerosoles, por lo que debe mantenerse una vigilancia activa del paciente con dificultad respiratoria para enviarlo precozmente a una Unidad de Cuidados Intensivos y no retrasar el procedimiento $^{27,34,35}$. Si fuese necesario, se deberá usar EPP completo: mascarilla N95, gafas y protección de contacto con guantes y delantal desechable siguiendo un protocolo estricto ${ }^{27}$. De ser perentoria la intubación endotraqueal, y que se pueda realizar la secuencia de intubación rápida, es muy importante realizar la preoxigenación con máscara de no reinhalación evitando la ventilación manual, en lo posible, y luego realizar el bloqueo neuromuscular con la medicación habitual (ver punto 14).

12. Apoyo ventilatorio: En caso de mayor deterioro del paciente con insuficiencia respiratoria o shock, el paciente requerirá ventilación asistida. La estrategia debe ser anticipatoria, de modo tal de enviar precozmente a una unidad de cuidados intensivos para que en las mejores condiciones se tome la decisión de qué tipo de asistencia ventilatoria se le indicará. Se ha planteado que, por la generación de aerosoles, debe preferirse ventilación mecánica invasiva (VM) por sobre la no Invasiva (VMNI), pero tomando las precauciones hay experiencias con éxito con VMNI en pacientes hospitalizados. En el escenario de urgencias no se recomienda su uso $^{36}$.

Como se mencionó, la VMNI tiene un mayor riesgo de generación de aerosoles que la ventilación mecánica invasiva, dado que existe un sistema de presurización que considera fugas intencionales, además de las producidas por la mala adaptación de las interfaces a la cara del paciente. Sin embargo, en casos con insuficiencia respiratoria hipoxémica moderada, con imposibilidad de un traslado precoz a una unidad de cuidados intensivo, en box cerrado idealmente con presión negativa, ésta puede ser útil, con monitorización frecuente, hasta el traslado del paciente. Se debe contar con un doble circuito o uno monorrama, pero con una pieza 
en Y más un filtro humedificador pasivo, HEPA, que permita además de termo-humedificar filtrar con alta eficiencia las partículas virales aerosolizadas. Las interfaces deben ser no ventiladas o con los portales exhalatorios ocluidas, que permitan la exhalación por un portal ad-hoc con el filtro ya mencionado.

No se aconseja realizar ventilación con bolsa-mascarilla y menos aconsejable aún con bolsa autoinflable. Si no es posible evitarlo, usar filtro de alta eficiencia e instalarlo justo después de la mascarilla realizando una ventilación a dos manos para lograr un muy buen selle y evitar fugas (técnica $\mathrm{V}-\mathrm{E})^{27,36,37}$.

13. Lugar de hospitalización: Según la gravedad que tengan los pacientes en urgencias, los riesgos determinados por la edad, asociación con otras enfermedades o uso de dispositivos especiales de apoyo que utilicen, se decidirá dónde será hospitalizado el paciente con COVID-19 (tabla 2) Las habitaciones de los hospitalizados por CoV2, deben ser individuales e idealmente con presión negativa. $\mathrm{Si}$ esto no es posible, se debe preferir hospitalizar en cohortes y siempre con distancias de al menos un metro de otra cama.

14. Paciente en paro cardiorrespiratorio (PCR): Se han publicado recientemente, las recomendaciones de la American Heart Association respecto a las maniobras de resucitación en un paciente sospechoso de COVID-19 en PCR $^{38}$. Lo primero es tener claro la pertinencia de reanimar según el pronóstico del paciente. Es prioritario, que los reanimadores estén con EPP antes de comenzar la reanimación y limitar las personas que participen en el procedimiento al mínimo necesario. Ventilar con bolsa-mascarilla con filtro y buen selle a dos manos si es estrictamente necesario. Una vez que se ha instalado el monitor desfibrilador, prepararse para intubar eligiendo al más experimentado, ojalá con videolaringoscopía, utilizando siempre tubo endotraqueal con cuff. Esta medida tiene la consideración de evitar al máximo la eliminación de aerosoles de virus y no seguir "bolseando" al paciente como lo hacemos en otros casos de PCR. Una vez intubado conectar a ventilación mecánica con filtro. Se aconseja minimizar al máximo las desconexiones del circuito. Hay que seguir estrictamente el protocolo de retiro del EPP y también para la desinfección del instrumental reutilizable ocupado.

\section{Discusión y conclusiones}

La infección por COV2 en niños, produce un cuadro la mayoría de las veces leve, que puede ser tratado en domicilio. El 95\% de los pacientes que consulten en el SU se irán al domicilio. Hay que tener especial cuidado con los niños menores de 1 año, recién nacidos contactos de sus madres con COVID-19, pacientes con enfermedades crónicas, con necesidades especiales en salud, sobre todo aquellos con dependencias tecnológicas para vivir, inmunodeficiencias y enfermedades oncológicas. Es vital, que los cuidadores del paciente tengan muy claro en qué consisten las medidas de aislamiento y de protección para el resto del núcleo familiar, cómo se tratará al niño y cuándo será necesario volver a urgencias.

La medida de tener dos áreas completamente separadas, una para los pacientes sospechosos de COVID-19 y otra para el resto de los pacientes, es clave para contener y evitar nuevos contagios.

Como hay un porcentaje alto de niños asintomáti-

Tabla 2. Unidad de hospitalización según características del paciente

\begin{tabular}{ll}
\hline Unidad de Hospitalización & Características del paciente \\
\hline Sala básica & Requerimientos $\leq 2$ l/min por bigotera estables hemodinámicamente \\
Intermedio & Requerimiento $>2$ l/min por bigotera \\
En esta unidad habrá más apoyo y una monitorización continua & Recién nacido sospechoso de COVID-19 \\
para vigilar estado del paciente & $\begin{array}{l}\text { Pacientes con apoyo ventilatorio crónico } \\
\text { Pacientes con patología de base con riesgo de enfermedad grave }\end{array}$ \\
Unidad de Cuidados Intensivos & Requerimientos altos de oxígeno \\
En esta unidad la vigilancia es permanente y se cuenta con el & Necesidad de ventilación mecánica y otras terapias de sustitución \\
mayor apoyo para el paciente grave y crítico & Necesidad de apoyo vasoactivo \\
& Compromiso de conciencia \\
& $\begin{array}{l}\text { Meningoencefalitis } \\
\text { Síndrome hemagofagocítico }\end{array}$ \\
& Falla multiorgánica \\
\hline
\end{tabular}


cos, el médico y todo el resto del personal del SU deben cumplir estrictamente con las medidas de EPP, inclusive en las áreas en que no se están viendo pacientes sospechosos de COVID-19.

Es muy importante fijar criterios claros de admisión, reconocer al paciente grave y crítico, sin retrasar las medidas de estabilización y terapia que requieran.

Los médicos que trabajamos en urgencias, somos la primera línea hospitalaria, debemos estar preparados para el gran desafío que significa organizar nuestras urgencias, tratar eficientemente a estos pacientes, y al mismo tiempo protegernos y proteger al resto del equipo del virus CoV2 que puede llegar a ser letal.

\section{Conflicto de intereses}

Los autores declaran no tener conflicto de intereses.

\section{Referencias}

1 Zhou P, Yang X, Wang X, et al. A pneumonia outbreak associated with a new coronavirus of probable bat origin. Nature. 2020;579(7798): 270-273. DOI: 10.1038/s41586-020-2012-7.

2 Comité de Prevención y Control de IAAS, Hospital Clínico UC-Christus y Clínica San Carlos de Apoquindo. Flujo de manejo de caso sospechosos de infección por coronavirus 2019- $\mathrm{nCoV}$ en Servicio de Urgencias Versión no 4 03.03.2020.

3 Sociedad Española de Urgencias de Pediatría. Recomendaciones SEUP por SARS-CoV-2 (Cuadro Clínico: COVID-19) Madrid; 2020 https://seup. org/pdf_public/SeupCOVID-19.pdf. Última visita 14 de abril de 2020.

4 NHS England. Redeploying your secondary care medical workforce safely [Internet]. National Health System. 2020; https://www.england.nhs.uk/coronavirus/ wp-content/uploads/sites/52/2020/03/ Redeploying-your-secondary-caremedical-workforce-safely_26-March.pdf. Última visita 4 de mayo de 2020.

5 Shen K, Yang Y, Wang T, et al. Diagnosis, treatment, and prevention of 2019 novel coronavirus infection in children: experts' consensus statement. World J Pediatr. 2020. DOI: 10.1007/s12519-020-00343-7.

6 Jones VG, Mills M, Suárez D, et al. COVID-19 and Kawasaki disease: novel virus and novel case. Hosp Pediatr. 2020; doi: 10.1542/hpeds.2020-0123; BMJ 2020;369:m1710; doi: 10.1136/bmj. $\mathrm{m} 1710$.

7 Ministerio de Salud, Consejo Asesor COVID-19 Chile. Estrategias para aumentar la detección de SARS-CoV-2 en Chile. Santiago de Chile 26 de abril de 2020; https://www.minsal.cl/consejoasesor-informa-sobre-nuevas-medidaspara-aumentar-la-deteccion-de-sars-cov2-en-chile/. Última visita 28 de abril de 2020.

8 Bartoszko J, Farooqi M, Alhazzani W, Loeb M. Medical Masks vs N95 Respirators for Preventing COVID-19 in Health Care Workers A Systematic Review and Meta-Analysis of Randomized Trials.
Influenza Other Respir Viruses. 2020 Apr 4. DOI: 10.1111/irv.12745.

9 Wang J, Zhou M, Liu F. Reasons for healthcare workers becoming infected with novel coronavirus disease 2019 (COVID-19) in China. J Hosp Infect. 2020 Mar 6. DOI: 10.1016/j. jhin.2020.03.002.

10 Colegio Médico de Chile, Sociedad Chilena de Infectología. Recomendaciones uso de elementos de protección personal (EPP) Para trabajadores de la salud, Santiago de Chile 2020; http:// www.colegiomedico.cl/wp-content/ uploads/2020/03/recomendacionesEPP-2.pdf. Última visita 14 de abril de 2020.

11 Guidance for the donning and doffing of Personal Protective Equipment (PPE) for COVID-19 2020;

https://www.youtube.com/ watch? $\mathrm{v}=\mathrm{bG6zISnenPg \& feature}=\mathrm{emb}$ logo. Última visita 4 de mayo de 2020.

12 Van Doremalen N, Bushmaker T, Morris D, et al. Aerosol and Surface Stability of SARS-CoV-2 as Compared with SARSCoV-1. N Engl J Med. 2020 Mar 17. DOI: 10.1056/NEJMc2004973.

13 de Souza T, Nadal J, Nogueira R, Pereira R, Brandao M. Clinical Manifestations of Children with COVID-19: A Systematic Review. MedRxiv [observaciones no publicadas] 2020. https://www.medrxiv. org/content/10.1101/2020.04.01.2004983 3v1. DOI: 10.1101/2020.04.01.20049833 útima visita 4 de abril de 2020 .

14 Dong Y, Mo X, Hu Y, et al. Epidemiology of COVID-19 Among Children in China. Pediatrics. 2020; e20200702. DOI: 10.1542/peds.2020-0702.

15 Castagnoli R, Votto M, Licari A, et al. Severe acute respiratory syndrome coronavirus 2 (SARS-CoV-2). Infection in children and Adolescent. A systematic Review. 2020. Acceso el 3 de mayo de 2020. Disponible en JAMA Pediatr. doi: 10.1001/jamapediatrics.2020.1467.

16 Zimmermann P, Curtis N. Coronavirus Infections in children including COVID-19. An overview of the epidemiology, Clinical features, diagnosis, treatment and prevention options in children. Pediatric Infectious Disease Journal 2020;39(5)355-68.

17 Ren X, Liu Y, Chen H, et al. Application and Optimization of RT-PCR in Diagnosis of SARS-CoV-2 Infection. MedRxiv [observaciones no publicadas] 2020; https://www.medrxiv.org/conten t/10.1101/2020.02.25.20027755v2 DOI: 10.1101/2020.02.25.20027755. Última visita 14 de abril de 2020 .

18 Zhi-Min Chen, Jun-Fen-Fu, Qiang $\mathrm{Shu}$, et al. Diagnosis and treatment recommendations for pediatric respiratory infections caused by novel coronavirus; htpp://doi.org/10.1007/ s1259-020-00345-53. Última visita 4 de mayo de 2020.

19 Documento de manejo clínico del paciente pediátrico y paciente de riesgo con infección por SARS-CoV-2.6/3/2020 revisado 16/4/2020 Sociedad española de Pediatría. https://www.aeped.es/noticias/ documento-manejo-clinico-pacientepediatrico-y-pacientes-riesgo-coninfeccion-por-sars-cov2. Última visita 3 de mayo de 2020.

20 Xiapxia L, Hui Du, Jingjing Z, et al. Correspondence. Sars-CoV-2 infection in Children. N Engl J Med 382; 17. DOI: 10.10.1056/NEJMc2005073.

21 Xia W, Shao J, Guo Y, Peng X, Li Z, Hu D. Clinical and CT features in pediatric patients with COVID-19 infection: Different points from adults. Pediatric Pulmonology. 2020;55:1169-74.

22 Duan Y, Zhu Y, Tang L, Qin J. CT features of novel coronavirus pneumonia (COVID-19) in children. Eur Radiol. 2020; https://doi.org/10.1007/s00330020-06860-3). Última visita 3 de mayo de 2020.

23 Sofia S, Boccatonda A, Montanari $\mathrm{M}$, Spampinato M, D’ardes D, Cocco G, et al. Thoracic ultrasound and SARS-COVID-19: a pictorial essay. Journal of Ultrasound. 2020; doi. org/10.1007/s40477-020-00458-7 última visita el 29 de abril de 2020.

24 Protocolos fase IV. Orientación de manejo de infección por nueva coronovirus (COVID-19) en domicilio, fase IV. Última visita el 3 de mayo de 2020. https://www. 
minsal.cl/wp-content/uploads/2020/04/ COVID-EN-DOMICILIO-CAA.pdf not be used for man say doctors and scientists. BMJ 2020; 368:m1086 doi: 10.1136/bmj.m1086.

26 Bialek S, Gierke R, Hughes M, McNamara L, Pilishvili T, Skoff T. Coronavirus Disease 2019 in Children - United States, February 12-April 2, 2020. MMWR Morb Mortal Wkly Rep. 2020 Apr 10;69(14):422-426. DOI: $10.15585 / \mathrm{mmwr}$. mm6914e4.

27 Health Protection Surveillance Center: Infection Prevention and Control Guidance for COVID-19. Aerosol generating procedures; https://www. hpsc.ie/a-z/respiratory/coronavirus/ novelcoronavirus/guidance/ infectionpreventionand controlguidance/. Última visita el 2 de mayo de 2020.

28 Whittle J, Pavlov I, Sacchetti A, Atwood C, Rosenberg M. Respiratory support for adult patients with COVID-19. JACEP Open. 2020; 1-7. DOI: 10.1002/ emp2.12071.

29 The National Heart, Lung, and Blood Institute Acute Respiratory Distress Syndrome (ARDS) Clinical Trials Network. Comparison of Two FluidManagement Strategies in Acute Lung Injury. N Engl J Med. 2006 Jun 15;354(24):2564-75. DOI: $10.1056 /$ NEJMoa062200.

30 Medical Resuscitation Committee from the Division of Emergency Medicine at Cincinnati Children's. Critical Airway Obstruction Cincinnati 2020; http:// mrc.pemcincinnati.com/wp-content/ uploads/2013/08/CAT-Algorithm-912Protected.pdf. Última visita 14 de abril de 2020.

31 Bhimraj A, Morgan R, Shumaker A,
Lavergne V, Chi-Chung Cheng V, Edwards K, et al. Infectious Diseases Society of America Guidelines on the Treatment and Management of Patients with COVID-19 [Internet]. 1st ed. Arlington, VA: IDSA 2020; https://www. idsociety.org/globalassets/idsa/practiceguidelines/covid-19/treatment/idsa-covid19-gl-tx-and-mgmt-v1.0.3.pdf. Última visita 14 de abril de 2020.

32 Fundación Epistemonikos. Revisión sistemática - Reporte preliminar. Antimaláricos para el tratamiento de COVID-19 [Internet]. 2nd ed. Santiago de Chile 2020; última visita 14 de abril de 2020 https://es.epistemonikos. cl/2020/03/31/revision-sistematicareporte-preliminar-antimalaricos-para-eltratamiento-de-covid-19/

33 American College of Radiology. ACR Recommendations for the use of Chest Radiography and Computed Tomography (CT) for Suspected COVID-19 Infection [Internet]. Acr.org. 2020 última visita 14 de abril de 2020. https://www.acr.org/ Advocacy-and-Economics/ACR-PositionStatements/Recommendations-for-ChestRadiography-and-CT-for-SuspectedCOVID19-Infection.

34 Medical Resuscitation Committee from the Division of Emergency Medicine at Cincinnati Children's. RSI checklist for Airborne Precautions. [Internet]. Cincinnati 2020; última visita 1 de mayo de $2020 \mathrm{http}: / / \mathrm{mrc}$.pemcincinnati. com/wp-content/uploads/2020/04/RSIChecklist-Airborne-Base-1-April-2020. pdf

35 Tran K, Cimon K, Severn M, PessoaSilva CL, Conly J. Aerosol Generating Procedures and Risk of Transmission of Acute Respiratory Infections to Healthcare Workers: A Systematic
Review. PLoS One. 2012;7(4):e35797. DOI: 10.1371/journal.pone.0035797.

36 Ong J, Chir MB, Tosoni A, Kim Y, Kisson N, Murthy S. Coronavirus Disease 2019 in Critically ill Children: A narrative review of the literature. Pediatric Critical Care Medicine 2020. Acceso 1 de mayo de 2020. Disponible en DOI: 10.1097/ PCC. 000000000000243 .

37 Kneyber M, Medina A, Modesto V, et al. Practice recommendations for the management of children proven COVID-19 infection from the Paesdiatric Mechanical ventilation Consensus Conference and the section respiratory failure from the European Society for Paediatric and Neonatal Intensive care (ESPNIC). 2020. Acceso 3 de mayo de 2020. Disponible en https://espniconline.org/News/Latest-News/Practicerecommendations-for-managingchildren-with-proven-or-suspectedCOVID-19.

38 Edelson D, Sasson C, Chan P, et al. Interim Guidance for Basic and Advanced Life Support in Adults, Children, and Neonates With Suspected or Confirmed COVID-19: From the Emergency Cardiovascular Care Committee and Get With the Guidelines ${ }^{\circledR}$-Resuscitation Adult and Pediatric Task Forces of the American Heart Association in Collaboration with the American Academy of Pediatrics, American Association for Respiratory Care, American College of Emergency Physicians, The Society of Critical Care Anesthesiologists, and American Society of Anesthesiologists: Supporting Organizations: American Association of Critical Care Nurses and National EMS. Physicians. Circulation. 2020 Apr 9. DOI: 10.1161/ CIRCULATIONAHA.120.047463. 